\title{
Determining the Envelope of Emergent Agent Behaviour via Architectural Transformation
}

\author{
Oswaldo Terán ${ }^{*}$, Bruce Edmonds ${ }^{*}$ and Steve Wallis ${ }^{*}$ \\ *Centre for Policy Modelling, Manchester Metropolitan University, \\ Aytoun Building, Aytoun Street, Manchester, M1 3GH, UK. \\ Tel. +441612476478 Fax. +441612476802 \\ \{o.teran, b.edmonds, s.wallis\} @mmu .ac.uk \\ tDepartment of Operation Research and Centre for Simulation \\ and Modelling, Universidad de Los Andes. Venezuela
}

\begin{abstract}
In this paper we propose a methodology to help analyse tendencies in MAS to complement those of simple inspection, Monte Carlo and syntactic proof. We suggest an architecture that allows an exhaustive model-based search of possible system trajectories in significant fragments of a MAS using forward inference. The idea is to identify tendencies, especially emergent tendencies, by automating the search through possible parameterisations of the model and the choices made by the agents. Subsequently, a proof of these tendencies could be attempted over all possible conditions using syntactic proof procedures. Additionally, we propose a computational procedure to help implement this. The strategy consists of: unencapsulating the MAS so as to reveal the maximum information about logical dependencies in the system. This information is maximised by splitting the transition rules by time intervals and some parameters. An example applying this procedure is exhibited which 'compiles' the rules into this form. In the example the exploration of possibilities is speeded up by a factor of 14 . This makes possible the complete exploration of model behaviour over a range of parameterisations and agent choices.
\end{abstract}

\section{Introduction: Understanding MAS}

MAS can (and frequently do) exhibit very complex behaviour - in this fact lies their promise but it also means that they can be difficult to understand and predict. Broadly there are two means by which we can seek to understand MAS: through design and through observation. Careful design procedures based on well-understood formal models of agent behaviour help us to understand the behaviour of individual agents and, in special cases, larger parts of MAS. However understanding the behaviour of interacting groups of autonomous agents by formal design methods has its limitations, and even the most carefully designed MAS can exhibit emergent behaviour unforeseen by its designers. This is hardly surprising as half the point of autonomous agents is that they should be able to deal with circumstances unforeseen by their designers.

Thus a second important way in which we can control MAS (after careful design) is by inspecting and analysing the behaviour of MAS in a post hoc manner, so that this can inform our future design and control of them. In other words, just like any software 
development environment, to effectively deploy MAS one needs both design and debugging tools. The most common methods of such post hoc analysis are: firstly, by detailed scenario analysis, where a single MAS trajectory at a time is examined and analysed and secondly, using a Monte Carlo approach where the MAS is repeatedly run and statistics collected about general trends over a sample of trajectories.

The scenario analysis provides the richest source of information, typically providing far more detail than the programmer can possibly cope with. It is also inherently contingent and it can be difficult to separate out what can be taken as representative behaviour and what is exceptional. After examining and interacting with several such runs of the system it is up to programmers to abstract an understanding of the MAS's behaviour using their intuition; the scenario analysis only conclusively demonstrates possibilities.

A Monte Carlo approach can be used to separate out the exceptional from the representative in some cases, but has a number of drawbacks including: the sort of behaviour one is investigating may not be satisfactorily summarised using numerical devices (for example in safety critical systems it may be insufficient to know that a certain parameter probably stays within an acceptable range, on would want to know it does); and the use of statistics inevitably involves the use of certain assumptions, which may not always be appropriate.

In this paper we discuss the use of a constraint-based search of possible models which can be deployed on significant subspaces of the total space of MAS possibilities. Like the Monte Carlo approach this can be seen as falling half-way between syntactic proof procedures and single scenario analyses. Unlike the Monte Carlo approach it produces definite answers to questions relative to the chosen subspace of possibilities - it can be seen as model-based proof w.r.t. subsets of the possibilities. It does not magically solve the problems in understanding all emergent MAS behaviour but is a useful addition to our menu of tools because it embodies a different trade-off between semantic richness and computational efficiency.

We will begin in section 2, by outlining the main idea. The implementational concerns of the technique, i.e. the proposed architecture for doing the constraint-based model search in a "hunt" of tendencies is described in section 3. Following this (section 4), we will give an example of applying this architecture. Then in section 5, we will compare this procedure with a couple of related approaches. In section 6 we briefly position this approach with respect to single simulation inspection and general theorem proving. Finally, some conclusions are made.

\section{Exploring the envelope of emergent MAS behaviour}

We want to be able to establish a more general type of knowledge of emergent behaviour than can be gained from the inspection of individual runs of a system. In particular we want to know whether a particular emergent behaviour is a necessary consequence of the system or merely a contingent one. Thus we propose to translate the system from an agent formulation into a form whereby the envelope of system possibilities can be determined, under a wider range of conditions. The target we have chosen is a constraintbased architecture: the MAS, modulo a range of parameterisations and nondeterministic agent choices, are translated into a set of positive constraints and the inference engine 
then searches for a model (i.e. a representation of a possible simulation in the original MAS with a particular parameterisation and set of choices) that satisfies these. This establishes the consistency of the positive constraints ${ }^{1}$. Postulated formulations of general emergent behaviour can be tested as to their necessity over the range of parameterisations and nondeterministic choices by negating them and adding them as a further constraint followed by getting the system to check that there is now no possible model.

The idea is to do this in a way which makes it possible to translate the MAS into the constraint-based one in an automatic or near automatic way without changing the meaning of the rules that make it up. In this way a user can program the system using the agentbased paradigm with all its advantages, inspect single runs of the system to gain an intuitive understanding of the system and then check the generality of this understanding for fragments of the system via this translation into a constraint-based architecture.

\section{Implementing a suitable constraint-based architecture}

The main goal of the programming strategy to be described is to increase the efficiency in terms of simulation time, thus making the constraint search possible. The improvements will be achieved by making the rules and states more context-specific. This enables the inference engine to exploit more information about the logical dependencies between rules and thus increase the efficiency. Thus this can be seen as a sort of 'practical compilation' process which undoes the agent encapsulation on an implemented MAS system in order to allow the more efficient exploration of its behaviour. In particular we split the transition rules into one per simulation period, and also by the initial parameters. This necessitates a dynamic way of building rules. This is done via a controller which generates the rules at the beginning of the simulation.

\subsection{Time Discrete Simulation Approach}

In synchronous simulations, time is taken as a discrete variable, given here as a set of positive numbers. In our case, we will call any of these numbers where the state variables are recalculated, a simulation time instant (STI) and the amplitude of the interval between two consecutive numbers, a simulation time step (STS). The transition function determines the state variables for STIs using the known values of the state variables in previous STIs. It is assumed that the state variables remain constant between two consecutive time instants.

In this architecture the structure of the simulated system is more than the one usually described in simulation formalisations (see for example Zeigler, 1976), e.g., it allows certain forms of structural change. A meta-agent as a controller in a MAS could guide not only quantitative changes, but also qualitative ones admitting its introduction into an evolutionary environment in a modular and transparent manner.

\footnotetext{
${ }^{1}$ Relative to the logic of the inference engine and range of parameters and choices allowed
} 


\subsection{Overview of the Architecture}

We implemented the proposed architecture in three parts, let us call them model, prover and meta-prover (we happen to have implemented these as agents but that is not important). The following illustrates this:

\begin{tabular}{|c|c|c|}
\hline \multicolumn{3}{|c|}{ model } \\
\hline $\begin{array}{l}\text { - General parameters. } \\
\text { - Initialisation } \\
\text { - Trial Parameters } \\
\text { - Choices. } \\
\text { - Calculations and } \\
\text { decisions. } \\
\text { - Report. }\end{array}$ & $\begin{array}{l}\quad \text { prover } \\
\text { Modules: } \\
\text { - Transition Rules (TR) } \\
\text { - Data-Rules (DR) } \\
\text { - Theorem checking (T) }\end{array}$ & $\begin{array}{l}\text { Meta-prover } \\
\text { Modules for writing rules for: } \\
\text { - State Transition (WTR). } \\
\text { - Calculate auxiliary data for } \\
\text { TR (WDR). } \\
\text { - Checking theorem (WT). }\end{array}$ \\
\hline $\begin{array}{l}\text { Part given the general } \\
\text { environment of the proof. }\end{array}$ & \multicolumn{2}{|c|}{$\begin{array}{l}\text { Part executing the proof ... it is } \\
\text { the instance where the proof is } \\
\text { done }\end{array}$} \\
\hline
\end{tabular}

Fig. 1. Illustration of the system parts.

\subsection{Program dynamics}

The system fires the rules in the following order:

1. model: initialising the environment for the proof (setting parameters, etc..)

2. meta-prover: creating and placing the transition rules in prover.

3. prover: carrying on the simulation using the transition rules and backtracking while a contradiction is found.

The program backtracks from a path once the conditions for the negated theorem are verified, then a new path with different choices is picked up. The next figure describes a transition step.

Prover just after the model has settled the parameters of the simulation and the initialisations $(i=$ 0 ), or after certain $S T I$, let's say $S T I_{i}$.
Meta-prover, writes on Prover the rules for period 1 , if it is the beginning of the simulation; or for period $S T I_{i+1}$ and using data from periods $1,2, \ldots S T I_{i}$, in other cases.

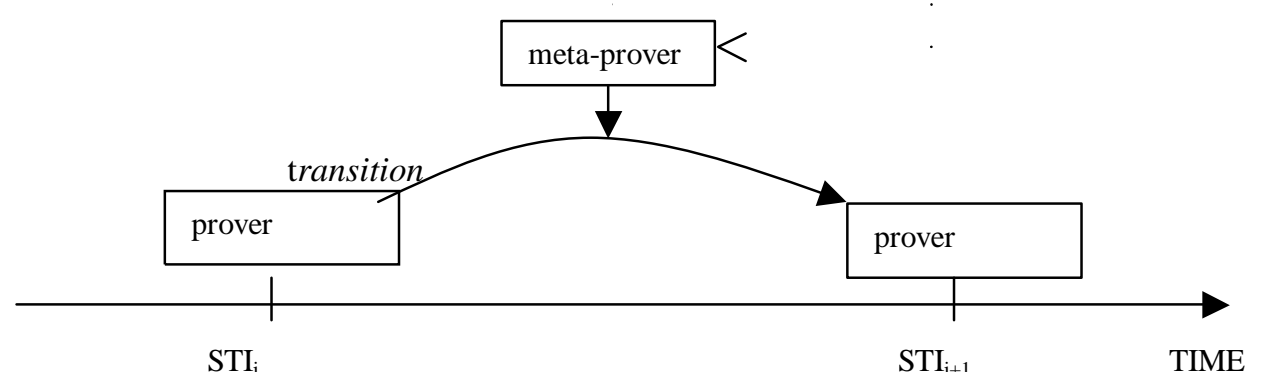

Fig. 2. State transition from $S T I_{\mathrm{i}}$ to $S T I_{i+1}$ 


\subsection{Description of System Modules}

General Parameters (GP). This will be placed in the model (see figure 1). Its task will be to set the general parameters of the simulation.

Initialising (I). This creates the entities (e.g. agents) given the static structure of the simulation and initialises the state variables for the first STI. It will be in model, as it is responsible for initialising parameters to be instantiated by meta-prover when writing the transition rules.

Trial Parameters (TP). To be placed in the model. Its task is to set up parameters to be fixed during one simulation trial. In general these are parameters for which the agents do not have to take decisions every STI (as for GP). They would be fixed before creating the transition rules.

Choices (CH). It will place alternatives for the choices the agents have every STI and the conditions under which each choice could be made. Choices will be mainly responsible for the splitting of the simulation and the rise of simulation branches.

Data Rules (DR), and Calculations and Decision rules (C\&D). The first module would contain the set of rules responsible for doing calculations required by the transition rules and which is worthy to keep in the database (they could evolve like the TR). The second one is a sort of function generating a numerical or a truth value as a result of consulting the database and usually consists of backward chaining rules.

Theorem (Constraints)(T). These are the conditions for checking the theorem. The theorem will be examined by a rule written by meta-prover in prover.

Reports $(\mathbf{R})$. The purpose of this module seems to be simple: to give the user outputs about what is going on in the dynamic of the simulation. This module will allows the user to know facts about the branch being tested as well as about branches already tested.

Transition Rules (TR). This is the set of rules will be context dependent and will include explicitly syntactical manipulation to make more straightforward the linking among them.

\subsection{Split of the rules: a source of efficiency}

A graphical illustration of the split procedure would be:

In forward chaining simulation the antecedent retrieves instance data from the past in order to generate data for the present (and maybe the future):

$$
\text { past facts } \rightarrow \text { present and future facts }
$$

Traditionally, the set of transition rules are implemented to be general for the whole simulation. A unique set of transition rules is used at any STI.

As the simulation evolves, the size of the database increases and the antecedents have to discriminate among a growing amount of data. At $S T I_{i}$, there would be data from (i-1) alternative days matching the antecedent. As the simulation evolves it becomes slower because of the discrimination the program has to carry out among this (linearly) growing amount of data. 


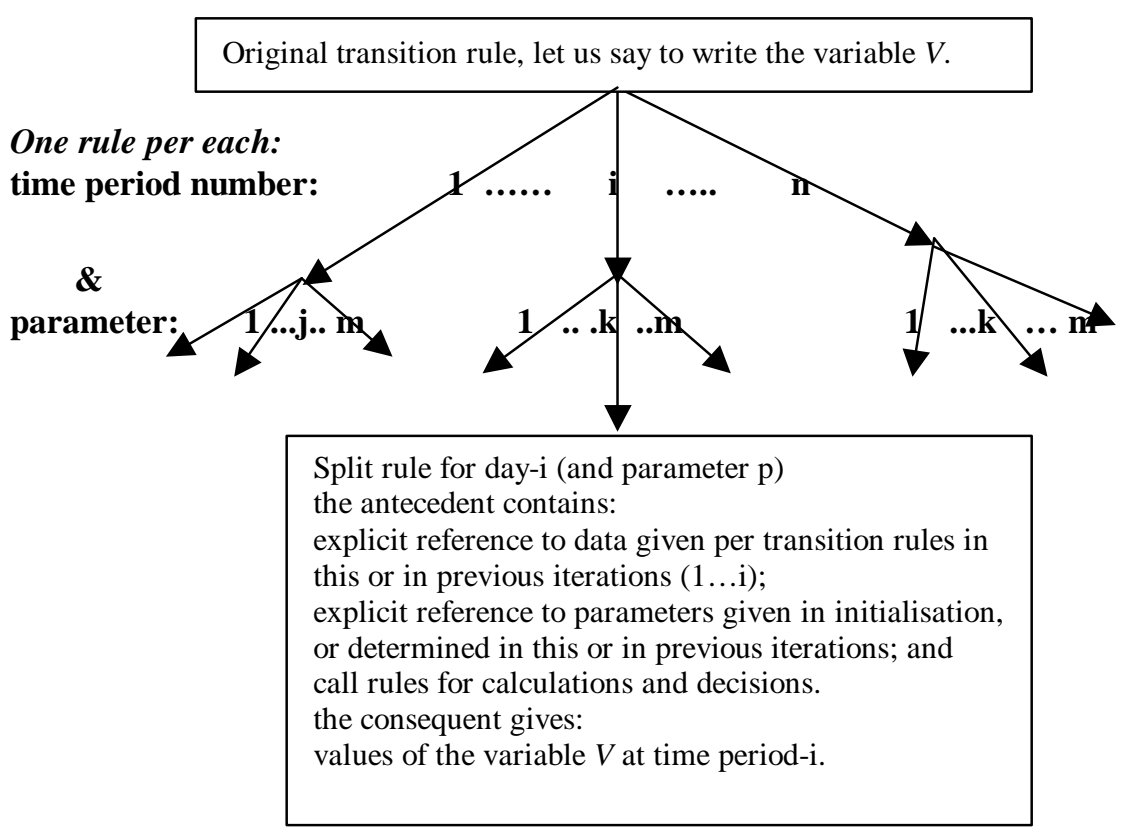

Fig. 3. Splitting of rules by time period and a combination of parameters

Using the proposed technique, we would write a transition rule for each simulation time. The specific data in the antecedent as well as in the consequent could be instanced. Where possible, a rule for each datum, the original rule will generate, would be written. This will be better illustrated in the example of the next section.

This technique represents a step forward in improving the efficiency of declarative programs, one could, in addition, make use of partitions and time levels. Partitions permit the system to order the rules to fire in an efficient way according to their dependencies. Time levels let us discriminate among data lasting differently. The splitting of rules lets us discriminate among the transition rules for different simulation times given a more specific instancing of data at any STI.

\subsection{Measuring the efficiency of the technique}

Comparing the two programs, the original and the one where the technique was implemented, in terms of the amount of data the program has to search into in order to check if a rule fires, we could have a rough idea about the increase in speed given by the technique.

While in the efficient program, each rule instances the specific data necessary to generate each datum at each STI, in the original one it has to discriminate among STIs and other not explicitly specified entities. For example, if there were three instances of 'producer', and the antecedent of a rule refers to 'producer', the rule has to discriminate among the three instances. This does not happen when using the technique. So, if there are $\mathrm{N}$ instances of any entities in certain rule, the technique speeds up the simulation in a factor of $\mathrm{N}$ when firing such a rule. Similarly, the technique speeds up the simulation by discriminating among STIs. 
The technique allows a speed by a factor of $N M / 2$. SDML already has facilities for discriminating among STIs, but their use is not convenient for the sort of simulation we are doing (exploring scenarios and/or proving) because of the difficulties for accessing data from any time step at any time. If we had used this facility in the original simulation model, it would have been speeded up by $M N(M-1) / 2$.

It is clear that the greater the number of entities in the simulation or the number of STIs, the larger the benefits the technique gives. We must notice that the speeding up of the simulation is only one aspect of the efficiency given by the technique.

\subsection{Translating a traditional MAS architecture into a model-exploration MAS architecture.}

Before splitting the rules the original MAS is reduced in a sort of unencapsulation of the hierarchy of agents into the architecture shown in figure 1. Additional variables must be added into predicates and functions in order to keep explicit the reference to the "owner" agent of any instance of a variable. This will facilitate the check for tendencies, the testing of the theorem and any other data manipulation. It is as if the agent where replaced by its rulebase, see figure 4.

In the original architecture, each agent has its own rulebase (RB) and database (DB). The agent's structure is given by its set $\langle\mathrm{RB}, \mathrm{DB}\rangle$ as well as by the structure of any subagents.

MAS Architecture:

each square represents an agent $(A)$ with its rulebase $(R B)$ and database (DB)

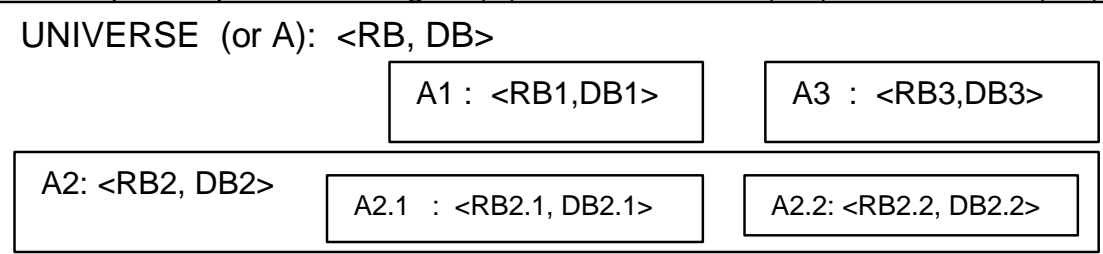

Logically partitioned: each square represents a partition

unencapsulation

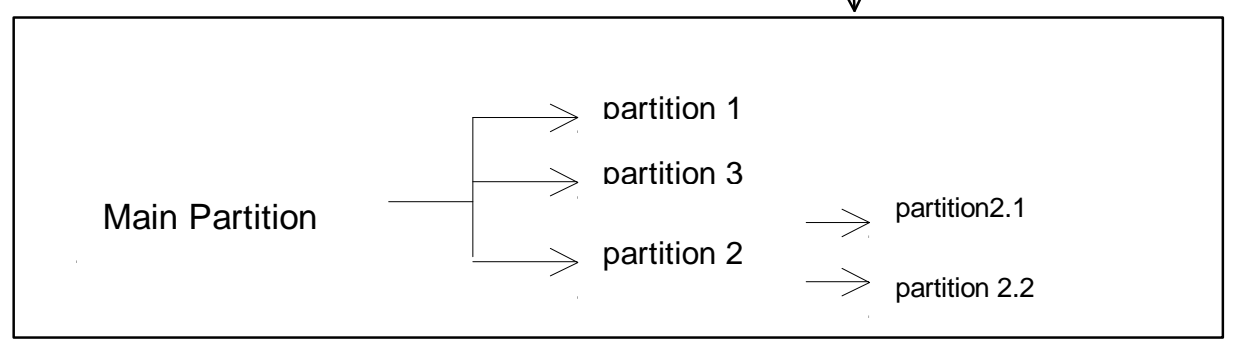

Fig. 4: Unencapsulating a MAS architecture

Using the technique, the initialisation of the static structure is accomplished by the module "Initialising", as explained above. The transition rules (dynamic structure) will be situated in the module "Transition Rules". There is still a hierarchy, both in the structure of the model and in the dynamics of the simulation - it is given by the precedence in the rulebase partition (figure 4). 
Now we turn to show a way of implementing the technique automatically. After adding variables to associate data with agents, the task is to write it modularly, as illustrated in figure 4. One of the key issues is to determine dependencies among rules and then choose appropriate data structures to allow the meta-prover to build the TR. A procedure to do it would be:

1. Identify parameters and entities for splitting (agents and/or objects) as well as the dependencies among rules. Look for a "general" description of the dependencies. E.g. a Producer's price at $S T I_{i}$ depends on Producers' sales and prices at $S T I_{i-1}$.

2. Create a list of references or links to each datum used in dependencies. Taking the previous example, a list containing the names of the clauses for prices and sales is created $\left(\left[\right.\right.$ Price $_{1}$, Price $_{2} \ldots$, Price $\left._{n}\right],\left[\right.$ Sale $_{1}$, Sale $_{2}, \ldots$, Sale $\left._{n}\right]\left(\right.$ Price $_{i}$, refers to price at $\left.S T I_{i}\right)$. This list could be also specified by producer, if necessary.

3. Initialise parameters (GP and TP) and data at $S T I_{1}$. It would be a task of module I (see above). It creates data used by module WTR at meta-prover and which are input for TR at $\mathrm{STI}_{2}$.

4. Provide the values for the choices the agents have.

5. Using these data structure and our knowledge about dependencies, we must be able to write the WTR, WDT, and WT at meta-prover. If TR at $S T I_{i}$ depend on data at $S T I_{i-1}$ then the list named in 2 . would allow to make such a reference automatically accessing the appropriate elements in the list.

6. Modules like R, C\&D are auxiliary and do not need special attention.

Constraints in the search are applied in different ways, for example when theorem is adapting (maybe relaxing conditions for a tendency) and as WTR and WDR take into account the past and present dynamic of the system (for instance, when restricting choices for the agents or objects, constraining the space of simulation paths).

\subsection{The platform used}

We implemented the systems described entirely within the SDML programming language ${ }^{2}$. Although this was primarily designed for single simulation studies, its assumption-based backtracking mechanism which automatically detects syntactic logical dependencies also allows its use as a fairly efficient constraint-based inference system. SDML also allows the use of "meta-agents", which can read and write the rules of other agents. Thus the use of SDML made the procedures described much easier to experiment with and made it almost trivial to preserve the meaning and effect of rules between architectures. The use of a tailor-made constraint-satisfaction engine could increase the effectiveness and range of the techniques described once a suitable translation were done, but this would make the translation more difficult to perform and verify.

\section{An example}

A simple system of producers and consumers, which was previously built in SDML and in the Theorem Prover OTTER, was rebuilt using the proposed modelling strategy. In the new model the exploration of possibilities is speeded up by a factor of 14 .

\footnotetext{
${ }^{2}$ Information about SDML can be found at http://www.cpm.mmu.ac.uk/sdml or (Moss et al., 1998)
} 
Some of the split transition rules were the ones for creating (per each STI) producers' prices and sales, consumers' demand and orders, warehouses' level and factories' production. Among the rules for auxiliary data split were the ones for calculating: totalorder and total-sales (a sum of the orders for all producers), total-order and total-sales per producer, and total-order and total-sales per consumer.

\subsection{Example of a split rule: Rule for prices}

This rule calculates a new price for each producer at each STI (which we called day), according to its own price and sales, and the price and sales of a chosen producer, at the immediately previous STI.

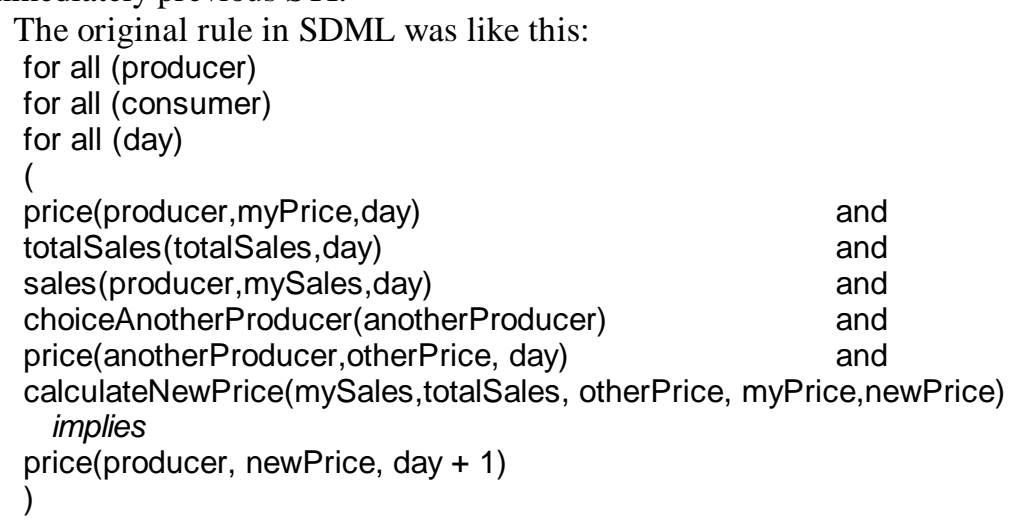

The new rule (in the efficient program) will be "broken" making explicit the values of prices and sales per each day.

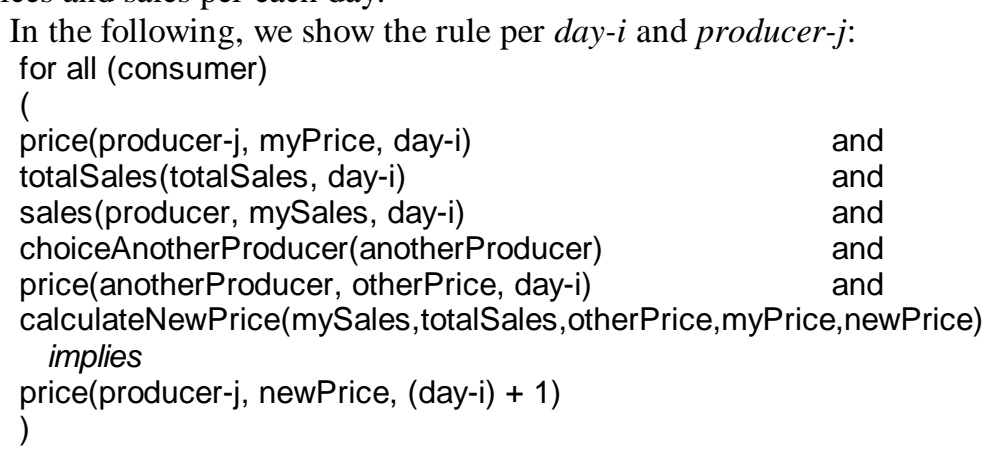

If the name of price is used to make explicit the day, the rule will have the following form. It is important to observe that only one instance of newPrice in the consequent is associated with only one transition rule and vice verse: 


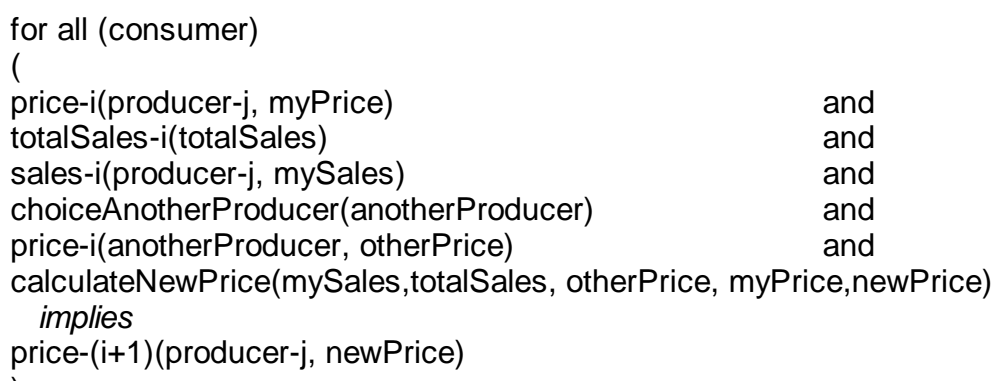

\subsection{What the technique enables}

In this example, we used the technique to prove that the size of the interval of prices (that is: biggest price - smaller price, each day) decreased over time during the first six time intervals over a range of 8 model parameterisations. An exponential decrease of this interval was observed in all the simulation paths. All the alternatives were tested for each day - a total of 32768 simulation trajectories. It was not possible to simulate beyond this number of days because of limitations imposed by computer memory. There was no restriction because of the simulation time, as the technique makes the simulation program quite fast - it had finished this search in 24 hours.

This technique is useful not only because of the speeding up of the simulation but also for its appropriateness when capturing and proving emergence. On one hand, it let us write the transition rules and the rule for testing the theorem at the beginning of the simulation in accordance to the tendency we want to prove. And, on the other hand, if the meta-prover is able to write the rules while the simulation is going on, it could adapt the original theorem we wanted to prove according to the results of the simulation. For example, if it is not possible to prove the original theorem then it could relax constraints and attempt to show that a more general theorem holds. Moreover, the technique could be implemented so that we have only to give the program hints related to the sort of proof we are interested in, then the meta-prover could adapt a set of hypotheses over time according to the simulation results. At best, such a procedure would find a hypothesis it could demonstrate and, at worst, such output could then be useful to guide subsequent experimentation.

\section{Some Other Approaches}

In OTTER (and similar Theorem Provers) the set of simulation rules and facts (atoms) is divided into two sets (this strategy is called support strategy) (McCune 1995):

One set with "support" and the other without it. The first one is place in a list called "SOS" and, the second one, in the list "USABLE". Data in USABLE is "ungrounded" in the sense that the rules would not fire unless at least one of the antecedents is taken from the SOS list. Data inferred using the rules in USABLE are placed in SOS when they are not redundant with the information previously contained in this list, and then used for generating new inferences. The criteria for efficiency are basically subsumption and weighting of clauses. 
Rules are usually fired in forward chaining but backward chaining rules and numerical manipulations are allowed in the constructs called "demodulators" (Wos, 1988).

In simulation strategies like event-driven simulation or partition of the space of rules, in declarative simulation, are used. The criteria for firing rules is well understood, and procedures like weighting and subsumption usually are not necessary. Additionally, redundant data could be avoided in MAS with a careful programming.

The advantages given for the weighting procedure in OTTER are yielded in MAS systems like SDML by procedures such as partitioning, where chaining of the rules allows firing the rules in an efficient order according to their dependencies.

Among other approaches for the practical proof of MAS properties, the more pertinent might be the case conducted by people working in DESIRE (Engelfriet et. al., 1998). They propose the hierarchical verification of MAS properties, and succeeded in doing this for a system.

However, their aim is limited to verification of the computational program - it is proved that the program behaves in the intended way. It does not include the more difficult task, which we try to address, of establishing general facts about the dynamics of a system when run or comparing them to the behaviour observed in other systems (Axtell et al., 1996).

\section{A sequence of architectures for modelling behaviour and proving theorems in MAS}

The architecture we describe could be used after single simulations have been run to suggest useful properties to test for. Subsequently one could employ a syntactically oriented architecture for proving those tendencies outright. Thus the proposed technique can be seen as falling in between inspecting single runs and syntactic theorem proving. This is illustrated in figure 5 .

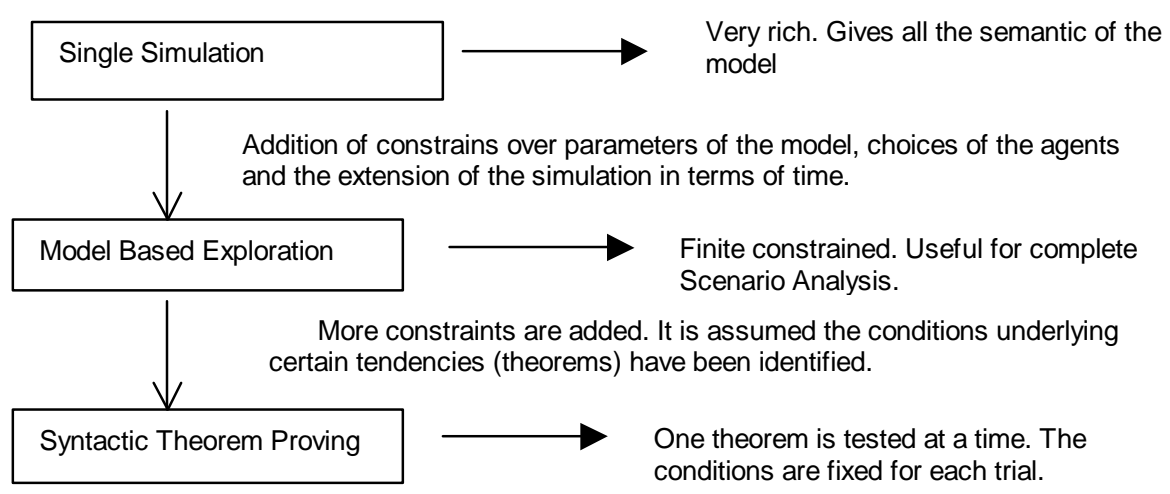

Fig. 5. Sequence of modelling architectures

The step to theorem proving from model-based exploration would involve a further translation step. The conditions established by experimenting with model-based exploration would need to be added to the MAS specification and all this translated into axioms for the theorem prover to work upon. The main aspects of the three architectures are summarised in Table 1, which is a comparison of these different approaches. 


\begin{tabular}{||c|l|l|l||}
\hline $\begin{array}{c}\text { Architecture: } \\
\text { Aspect: }\end{array}$ & \multicolumn{1}{|c|}{$\begin{array}{c}\text { SCENARIO } \\
\text { ANALYSIS }\end{array}$} & $\begin{array}{c}\text { MODEL- } \\
\text { EXPLORATION }\end{array}$ & \multicolumn{1}{|c||}{$\begin{array}{c}\text { SYNTACTIC } \\
\text { PROOF }\end{array}$} \\
\hline $\begin{array}{c}\text { Typical } \\
\text { paradigm }\end{array}$ & Imperative. & Constraint & Declarative \\
\hline $\begin{array}{c}\text { Typical } \\
\text { deduction } \\
\text { system }\end{array}$ & $\begin{array}{l}\text { (Forward } \\
\text { Chaining) }\end{array}$ & $\begin{array}{l}\text { Forward Chaining } \\
\text { using efficient } \\
\text { backtracking }\end{array}$ & $\begin{array}{l}\text { Backward chaining } \\
\text { or resolution-based }\end{array}$ \\
\hline $\begin{array}{c}\text { Nature of the } \\
\text { manipulations }\end{array}$ & Possible Semantic & Range of Semantics & Syntactic \\
\hline Limitations & $\begin{array}{l}\text { Not constrained. } \\
\text { - Very rich. Too } \\
\text { much information } \\
\text { could mislead }\end{array}$ & $\begin{array}{l}\text { - Finite constrained. } \\
\text { Still quite rich. } \\
\text { - Suitable for } \\
\text { Scenario Analysis. }\end{array}$ & $\begin{array}{l}\text {-Constrained. } \\
\text { - Valuable for } \\
\text { proving specific } \\
\text { tendencies. }\end{array}$ \\
\hline Search style. & $\begin{array}{l}\text { Attempts to } \\
\text { explore all } \\
\text { simulation paths. }\end{array}$ & $\begin{array}{l}\text { Limits the search by } \\
\text { constraining the } \\
\text { range of parameters } \\
\text { and agent choices }\end{array}$ & $\begin{array}{l}\text { Can be efficient in } \\
\text { suitably constrained } \\
\text { cases, typically } \\
\text { impractical }\end{array}$ \\
\hline \hline
\end{tabular}

Table 1. A Comparison of Architectures

\section{Conclusion}

The proposed methodology is as follows: firstly, identify candidate emergent tendencies by inspection of single runs; secondly, explore and check these using the techniques of constraint-based model-search in significant fragments of the MAS; and finally, attempt to prove theorems of these tendencies using syntactic proof procedures. This methodology is oriented towards identifying interesting tendencies and emergence in MAS an area little explored but of considerable importance.

In addition, we have proposed a framework for improving the efficiency of MAS to enable the second of these. It has been implemented in an ideal example, resulting in a significant increase in the speed of the program. However, the notions are valid independently of the example and could be implemented in many different systems. In summary, the strategy consists of: unencapsulating the MAS system to allow the maximum amount of dependency information to be exploited; partitioning of the space of rules and splitting of transition rules by STI and some parameters, using the appropriate modularity of the simulation program, and specially initialising parameters and choices.

The technique perhaps presages a time when programmers routinely translate their systems between different architectures for different purposes, just as a procedural programmer may work with a semi-interpreted program for debugging and a compiled and optimised form for distribution. In the case of agent technology we have identified three architectures which offer different trade-offs and facilities, being able to 
automatically or semi-automatically translate between these would bring substantial benefits.

Acknowledgements. SDML has been developed in VisualWorks 2.5.2, the Smalltalk-80 environment produced by ObjectShare. Free distribution of SDML for use in academic research is made possible by the sponsorship of ObjectShare (UK) Ltd. The research reported here was funded by CONICIT (the Venezuelan Governmental Organisation for promoting Science), by the University of Los Andes, and by the Faculty of Management and Business, Manchester Metropolitan University.

\section{References}

Axtell, R., R. Axelrod, J. M. Epstein, and M. D. Cohen (1996), “Aligning Simulation Models: A Case of Study and Results", Computational Mathematical Organization Theory, 1(2), pp. 123141.

Engelfriet, J., C. Jonker and J. Treur (1998) "Compositional Verification of Multi-Agent Systems in Temporal Multi-Epistemic Logic”, Artificial Intelligence Group, Vrije Universiteit Amsterdam, The Netherlands.

McCune, W. (1995), OTTER 3.0 Reference Manual Guide, Argonne National Laboratory, Argonne, Illinois.

Moss, S., H. Gaylard, S. Wallis, B. Edmonds (1998), "SDML: A Multi-Agent Language for Organizational Modelling", Computational Mathematical Organization Theory, 4(1), 43-69.

Wos, L. (1988), Automated Reasoning: 33 Basic Research Problems, Prentice Hall, New Jersey, USA.

Zeigler, B. (1976), Theory of Modelling and Simulation, Robert E. Krieger Publishing Company, Malabar, Fl, USA. 